\title{
An Automated Draft Report Generator for Peripheral Blood Smear Examinations Based on Complete Blood Count Parameters
}

Young-gon Kim, M.D. ${ }^{1}$, Jung Ah Kwon, M.D. ${ }^{1}$, Yeonsook Moon, M.D. ${ }^{2}$, Seong Jun Park, M.D. ${ }^{1}$, Sangwook Kim, M.D. ${ }^{1}$, Hyun-A Lee, M.T. ${ }^{1}$, Sun-Young Ko, M.D. ${ }^{1}$, Eun-Ah Chang, M.D. ${ }^{1}$, Myung-Hyun Nam, M.D. ${ }^{1}$, Chae Seung Lim, M.D. ${ }^{1}$, and Soo-Young Yoon (i), M.D., Ph.D. ${ }^{1}$

${ }^{1}$ Department of Laboratory Medicine, Korea University College of Medicine, Seoul, Korea; ${ }^{2}$ Department of Laboratory Medicine, Inha University School of Medicine, Incheon, Korea

Background: Complete blood count $(\mathrm{CBC})$ results play an important role in peripheral blood smear (PBS) examinations. Many descriptions in PBS reports may simply be translated from CBC parameters. We developed a computer program that automatically generates a PBS draft report based on CBC parameters and age- and sex-matched reference ranges.

Methods: The Java programming language was used to develop a computer program that supports a graphical user interface. Four hematology analyzers from three different laboratories were tested: Sysmex XE-5000 (Sysmex, Kobe, Japan), Sysmex XN-9000 (Sysmex), DxH800 (Beckman Coulter, Brea, CA, USA), and ADVIA 2120i (Siemens Healthcare Diagnostics, Eschborn, Germany). Input data files containing $862 \mathrm{CBC}$ results were generated from hematology analyzers, middlewares, or laboratory information systems. The draft reports were compared with the content of input data files.

Results: We developed a computer program that reads $\mathrm{CBC}$ results from a data file and automatically writes a draft PBS report. Age- and sex-matched reference ranges can be automatically applied. After examining PBS, users can modify the draft report based on microscopic findings. Recommendations such as suggestions for further evaluations are also provided based on morphological findings, and they can be modified by users. The program was compatible with all four hematology analyzers tested.

Conclusions: Our program is expected to reduce the time required to manually incorporate $\mathrm{CBC}$ results into $\mathrm{PBS}$ reports. Systematic inclusion of $\mathrm{CBC}$ results could help improve the reliability and sensitivity of PBS examinations.

Key Words: Complete blood count, Peripheral blood smear, Draft report generator
Received: December 4, 2017

Revision received: February 1, 2018

Accepted: June 26, 2018

Corresponding author: Soo-Young Yoon (iD) https://orcid.org/0000-0002-2302-3825 Department of Laboratory Medicine, Korea

University College of Medicine, 148

Gurodong-ro, Guro-gu, Seoul 08308, Korea

Tel: +82-2-2626-3246

Fax: +82-2-2626-1465

E-mail: labmd@korea.ac.kr

\begin{abstract}
() Korean Society for Laboratory Medicine
This is an Open Access article distributed under the terms of the Creative Commons Attribution Non-Commercial License (http://creativecommons.org/licenses/by-nc/4.0) which permits unrestricted non-commercial use, distribution, and reproduction in any medium, provided the original work is properly cited.
\end{abstract}

\section{INTRODUCTION}

The peripheral blood smear (PBS) examination has direct diagnostic utility for certain groups of hematological and infectious diseases, such as leukemia, hemolytic anemia, and malaria, while providing adjunctive information to complete blood count
(CBC) results. PBS examinations are usually requested by physicians in response to perceived clinical features or to abnormalities in the $\mathrm{CBC}$ results [1]. When examining a PBS, CBC results play an important role as an interpretation guide; currently, sophisticated automated hematology analyzers provide various parameters and flags based on multiple technologies, including 
flow cytometry [2]. In order to derive maximal information from a PBS, these parameters should be considered and incorporated into PBS reports.

Many descriptions in PBS reports may have simply been interpreted from the $\mathrm{CBC}$ parameters without actually examining blood smears. For example, the description "microcytic hypochromic anemia with anisocytosis" could be written prior to examining the blood smears, based on CBC parameters such as hemoglobin, mean corpuscular volume (MCV), mean corpuscular hemoglobin $(\mathrm{MCH})$, mean corpuscular hemoglobin concentration (MCHC), and red blood cell distribution width (RDW). Likewise, "mild leukocytosis with neutrophilia" or "mild thrombocytosis" can also be written based on instrument-generated CBC parameters such as white blood cell (WBC) count, platelet count, and the percentage of neutrophils. According to the International Council for Standardization in Haematology (ICSH) [3], for some red blood cell (RBC) abnormalities, interpretation of the parameters produced by hematology analyzers may be more accurate and less subjective than grading by examining PBSs. Similarly, modern hematology analyzers provide accurate WBC differentials, which should be reported for patients with normal cell populations [3, 4].

In many clinical laboratories, hematopathologists spend a considerable amount of time reviewing and summarizing all the $\mathrm{CBC}$ parameters when preparing for PBS reports. For pediatric patients, especially for neonates, this process takes more time because their reference ranges are different from those of adults [5-7].

We aimed to optimize this part of the process by developing a computer program that automatically generates a PBS examination draft report based on CBC parameters. We also aimed to make our program aware of age- and sex-matched reference ranges and apply them when constructing the draft reports.

\section{METHODS}

The study was approved by the institutional review board of Ko- rea University Medical Center (KUMC; IRB number 2018GR 0119), Seoul, Korea. Initially, we used Sysmex XE-5000 (Sysmex, Kobe, Japan) as the hematology analyzer. As CBC results from Sysmex hematology analyzers can be saved into a Microsoft Excel file or comma-separated values (csv) file, we used that file as the input for our program. Once the initial version of our program had been implemented and tested, we modified our program to support other types of hematology analyzers. Our program was developed between December 2016 and June 2017. During this period, we tested four hematology analyzersSysmex XE-5000, Sysmex XN-9000 (Sysmex), DxH800 (Beckman Coulter, Brea, CA, USA), and ADVIA 2120i (Siemens Healthcare Diagnostics, Eschborn, Germany)—from three different hospitals: KUMC Guro Hospital (Seoul), KUMC Ansan Hospital (Ansan), and Inha University Hospital (Incheon; Table 1).

Data files could be generated directly by hematology analyzers, as well as by middlewares or laboratory information systems (LIS) depending on the information system architecture of the laboratories. For DxH8OO installed at KUMC Guro Hospital, HemLink, which is a middleware provided by Beckman Coulter, was used to generate data files. HemLink can communicate with the DxH8OO through a local network and can generate data files containing $\mathrm{CBC}$ results. We installed our program on a computer already equipped with HemLink, so that users could generate a data file from HemLink and open it instantly on the same computer without the need for a file transfer. For ADVIA 2120i, data files could not be directly generated from the hematology analyzer or middleware. Instead, the CBC parameters and flags were first uploaded into the LIS and then downloaded from the LIS as an Excel file.

To develop a graphical user interface (GUI)-based computer program, we used the Java programming language (Version 1.8) and its Swing components. Eclipse with WindowBuilder was used as the integrated development environment.

The draft report generated by our program was validated by comparing it manually with the content of input excel files. The number of cases utilized for testing was 460, 150, 153, and 99

Table 1. Hematology analyzers tested

\begin{tabular}{lllll}
\hline Model & \multicolumn{1}{c}{ Manufacturer } & Data file type & Data file generation & Location \\
\hline XE-5000 & Sysmex, Kobe, Japan & xlsx & Instrument & KUMC Guro Hospital \\
XE-5000 & Sysmex, Kobe, Japan & csV & Instrument & KUMC Ansan Hospital \\
XN-9000 & Sysmex, Kobe, Japan & xlsx & Instrument & KUMC Ansan Hospital \\
DXH800 & Beckman Coulter, Brea, CA, USA & xls & Middleware (HemLink) & KUMC Guro Hospital \\
ADVIA 2120i & Siemens Healthcare Diagnostics, Eschborn, Germany & xlsx & LIS & Inha University Hospital \\
\hline
\end{tabular}

Abbreviations: cSV, comma separated values; KUMC, Korea University Medical Center; LIS, laboratory information system. 


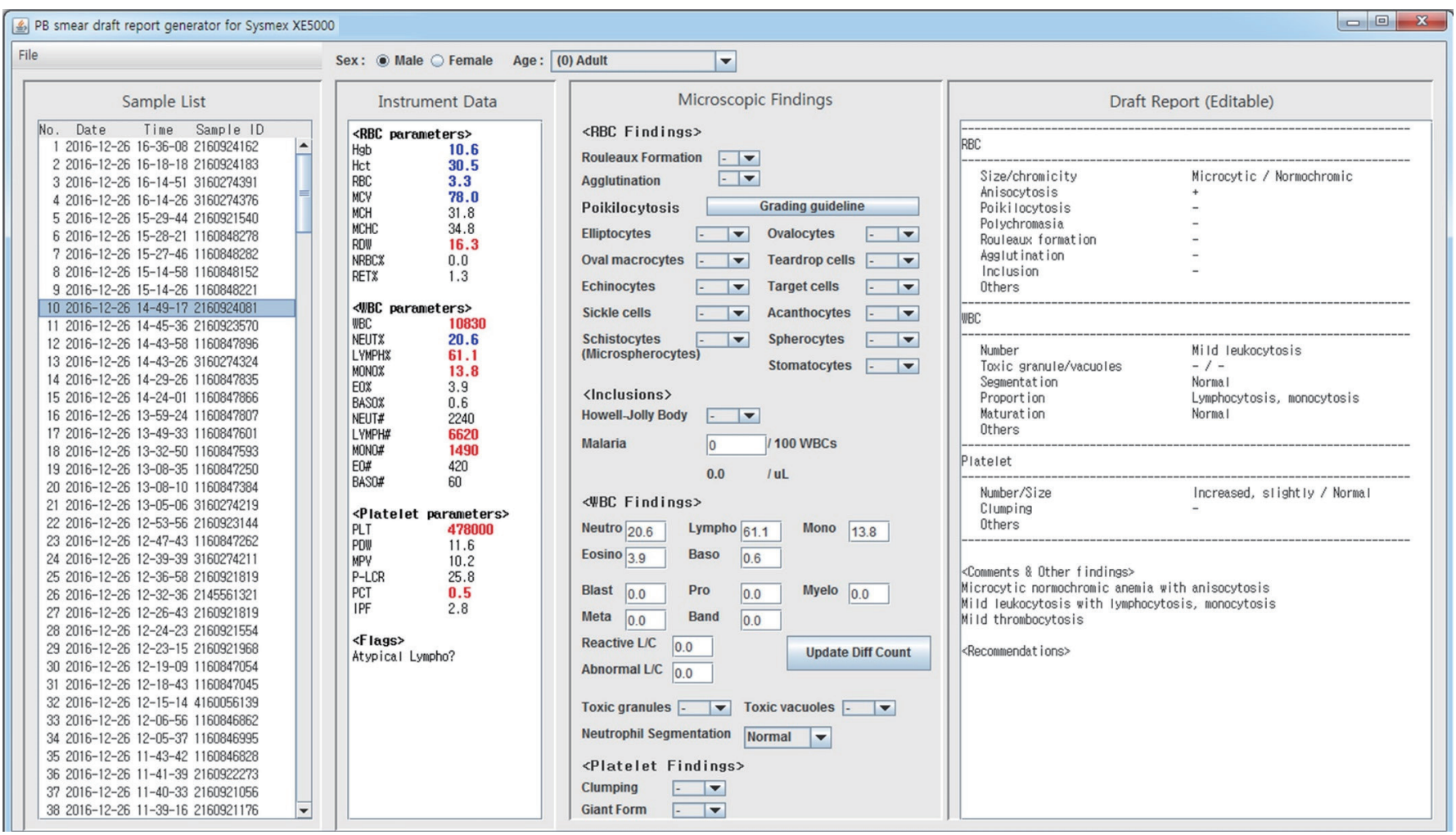

Fig. 1. Draft report: one sample is selected from the Sample List (left, shaded), and instantly, the Instrument Data panel is auto populated with complete blood count parameters. A draft report is prepared in the Draft Report panel. The Microscopic Findings panel remains in its initial state at this point. Age- and sex-matched reference ranges can be applied, if age and sex are manually selected (at the top of the screen). In this example, the Excel data file was generated from Sysmex XE-5000 (Sysmex, Kobe, Japan).

for Sysmex XE-5000, Sysmex XN-9000, DxH800, and ADVIA 2120i, respectively. Whenever an erroneous interpretation was found, our program was modified to correct that error. This process was repeated until all cases were interpreted correctly.

\section{RESULTS}

Our program consists of four separate panels: Sample List, Instrument Data, Microscopic Findings, and Draft Report (Fig. 1). Users can open a data file using the File tab on the menu bar. When the data file is initially loaded, all sample IDs contained in the data file are listed in the Sample List panel. When one of the samples from the Sample List is selected, CBC parameters and flags for that sample are listed in the Instrument Data panel, and at the same time, a draft report is automatically prepared in the Draft Report panel. Fig. 1 presents a snapshot of our program at this point. In this example, patient information such as age and sex is not stored in the data file. However, age- and sex-matched reference ranges can be applied when writing the draft report if age and sex are manually selected (Fig. 1).
The draft report is plain text that is editable at any time. It can be manually edited after examining the PBS; however, for routinely used expressions, tools are provided on the Microscopic Findings panel. Fig. 2 shows a snapshot of our program after applying the microscopic findings to the Microscopic Findings panel. Drop-down lists for describing rouleaux formation, agglutination, and poikilocytosis have been prepared. These are designed according to the ICSH guidelines, which recommend a two-tier reporting system (i.e., ++ and +++ ) to report only clinically significant results [3]. For example, in Fig. 2, the grading scale for elliptocytes includes only -, ++, and +++. According to the guidelines, the use of $+/$ - and + is reserved only for describing schistocytes, whose observation has clinical significance even at small numbers [3]. To aid users in grading poikilocytosis, the Morphology Grading Table from the ICSH guidelines [3] is linked to the "Grading guideline" button.

Textfields for manual differential counts are also provided. Initially, they contain the instrument-generated differential counts (Fig. 1) and can be modified after a manual differential count, if required. In our example, the "Atypical Lympho?" flag sug- 


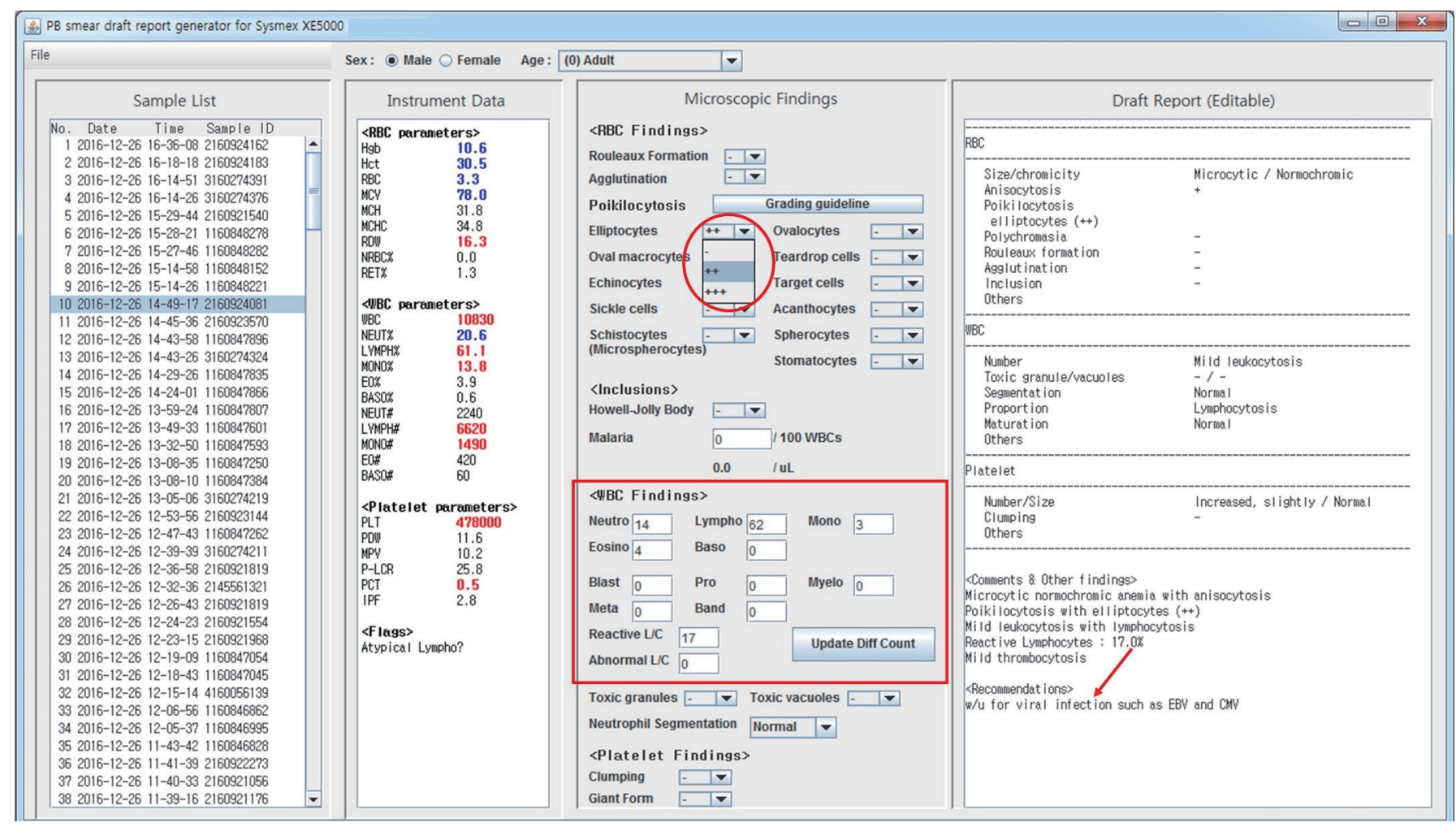

Fig. 2. Final report: A manual differential count was performed, and the results are typed into the Microscopic Findings panel (red box). All changes made in the Microscopic Findings (in this case, differential counts and poikilocytosis) are automatically reflected in the Draft Report. Work-up (w/u) for Epstein-Barr virus (EBV) and cytomegalovirus (CMV) markers is recommended, owing to the increased reactive lymphocyte count (arrow). The grading scale for poikilocytosis follows the International Council for Standardization in Haematology guidelines (red circle, -, ++, and +++ for elliptocytes) [3]. In this example, the Excel data file was generated from Sysmex XE-5000 (Sysmex, Kobe, Japan).

Table 2. Recommendations automatically generated according to morphological findings

\begin{tabular}{|c|c|}
\hline Finding & Recommendation \\
\hline Schistocytes, spherocytes & $\begin{array}{l}\text { Work-up for hemolysis (LDH, DAT, haptoglobin, } \\
\text { plasma Hb, etc.). }\end{array}$ \\
\hline Rouleaux formation & Serum and urine PEP/IFE, if clinically indicated. \\
\hline Blast & $\begin{array}{l}\text { CBC \& PBS follow up, BM examination if } \\
\text { clinically indicated. }\end{array}$ \\
\hline Abnormal lymphocytes & $\begin{array}{l}\text { CBC \& PBS follow up, BM examination if } \\
\text { clinically indicated. }\end{array}$ \\
\hline Reactive lymphocytes (>10\%) & $\begin{array}{l}\text { Work-up for viral infection such as EBV and } \\
\text { CMV. }\end{array}$ \\
\hline Platelet clumping & $\begin{array}{l}\text { Rule out EDTA-induced } \\
\text { pseudothrombocytopenia. } \\
\text { Resampling using Heparin or Citrate tube is } \\
\text { recommended. }\end{array}$ \\
\hline
\end{tabular}

Abbreviations: LDH, lactose dehydrogenase; DAT, direct antiglobulin test; PEP, protein electrophoresis; CBC, complete blood count; PBS, peripheral blood smear; IFE, immunofixation electrophoresis; BM, bone marrow; EBV, Epstein-Barr virus; CMV, cytomegalovirus; EDTA, ethylenediaminetetraacetic acid. gested morphological abnormalities in the lymphocytes, and the consequent manual differential count revealed $17 \%$ reactive lymphocytes. In Fig. 2, results of the manual differential count were typed into the textfields, and the draft report was modified automatically to reflect the new differential counts. To describe morphological abnormalities in lymphocytes, we followed the ICSH guidelines [3], which recommend using the term "reactive lymphocyte" to describe lymphocytes with a benign etiology and "abnormal lymphocyte" for those with a suspected malignant or clonal etiology.

All changes made in the Microscopic Findings panel are instantly reflected in the report. In our example, a work-up for viral markers is recommended because of the increased reactive lymphocyte count. Other recommendations provided according to the morphological findings are listed in Table 2. These are only suggestions, and users can take the final decision on whether to include them in the report.

Fig. 3 shows how sex- and age-matched reference ranges are automatically applied into draft reports when the input file con- 


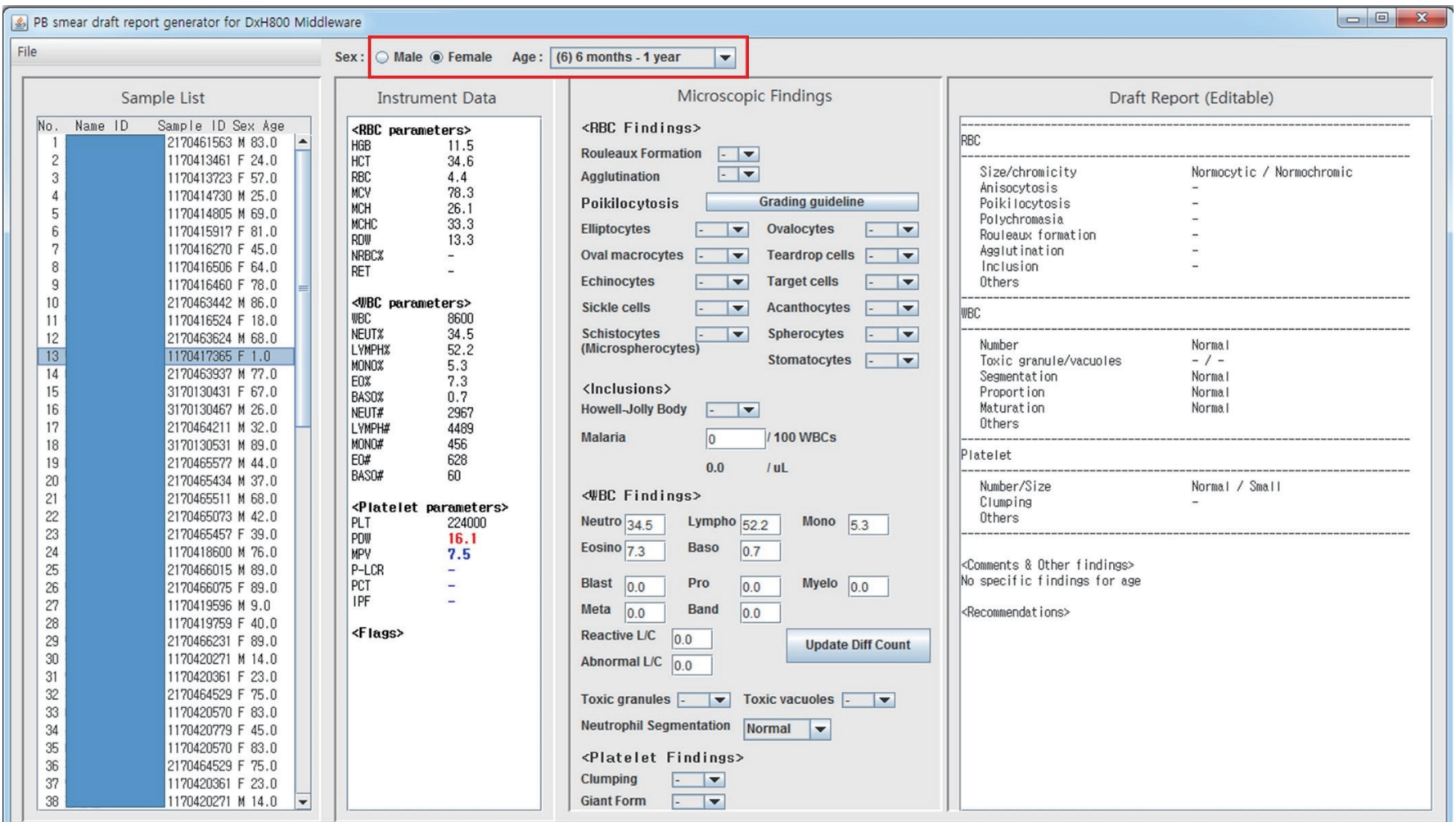

Fig. 3. In this example, the input data file contains patient information, such as sex and age, enabling automatic adjustment of sex and age options of the program (red box). In this case, the patient is one year old. The hemoglobin, mean corpuscular volume (MCV), and white blood cell (WBC) differential counts would have indicated microcytic anemia and relative lymphocytosis had adult reference ranges been applied. However, they are interpreted as normal, following the age-specific reference ranges. In this example, the Excel data file was generated from HemLink, a middleware for Beckman Coulter hematology analyzers.

tains the age and sex of the patients. The age is selected as adult by default when the age is not contained in the input data file. As of writing this article, our program, installed at KUMC Guro Hospital, is being utilized in every PBS examination together with a DxH800 and its middleware HemLink.

\section{DISCUSSION}

With advances in information technology, many pathology report generation tools have been developed. Synoptic reporting systems for bone marrow samples and lymphoid samples were developed and described by Murari et al [8] and Mohanty et al [9], respectively. A Web-based synoptic reporting program for PBS examinations has been described by Jaso et al [10]; however, the automatic incorporation of CBC parameters was not considered. These synoptic reporting systems improve efficiency, reduce turnaround time, and decrease reporting errors; the reports they generate enable quicker access to desired information and improved communication [8, 9]. We expect that our program will afford the same advantages, as we also provide a structured way of generating reports using predefined constructs.

Despite the ICSH recommendations, various formats are used for writing PBS reports, and efforts have been made to create a standard format $[3,11]$. Currently, the report generated by our program is based on the format used in KUMC Guro Hospital. Should a standardized reporting format be established in the future, even if it is not a global standard, our program can be easily modified to comply with it and can be used to facilitate the application of the new standard.

We expect that integration of our program with middlewares of hematology analyzers or with an LIS can further simplify the process. The cost of integration would vary depending on the target system; however, the fact that our program is written in Java, which is a platform-independent programming language, offers a definite advantage in case of integration.

Our program is best suited for laboratories that use hematology analyzer middleware. If our program is installed on the per- 
sonal computer on which the middleware is installed, the data file for our program can be generated from the middleware and can then be instantly opened in our program without the need for a file transfer. Moreover, the histograms and scattergrams that cannot be viewed from our program can be viewed on the same computer if the middleware is installed.

In many laboratories that do not use hematology analyzer middlewares, $\mathrm{CBC}$ results are printed out on paper and delivered to hematopathologists for PBS examinations. These printouts can be replaced by a data file if our program is used. Our program can still benefit the process because transferring a data file should be easier than printing, and the efforts involved in file transfer are compensated by an automated, systematic summary of the $\mathrm{CBC}$ results.

When patient information, such as age and sex, are available in the data files, age- and sex-matched reference ranges can be conveniently applied. Age-matched reference ranges can be obtained from multiple sources [6, 7, 12, 13], and their values do not differ significantly. We mainly followed the Mayo Medical Laboratories [7] values for building age- and sex-matched reference ranges, as well as Proytcheva [6] and Nathan and Oski [12].

One limitation of our work is that the histograms and scattergrams cannot be saved in data files and thus cannot be viewed from our program directly. The histograms and scattergrams can be viewed directly from the hematology analyzers or from middlewares or result printouts. However, the examination of histograms and scattergrams is not necessary for all PBS cases, and the existence of abnormalities in histograms or scattergrams can be identified by flags. CBC result printouts can be requested for cases, in which an examination of the histograms or scattergrams is deemed necessary. To view the histograms and scattergrams directly from our program, some level of integration between our program and the hematology analyzers or middlewares is necessary. Another limitation of our study is that we did not measure the time saved from manually incorporating CBS parameters into PBS reports.

In conclusion, our program is compatible with four different hematology analyzers from three different laboratories. It is expected to reduce the time required for manually incorporating CBC parameters into PBS reports while following age- and sexmatched reference ranges. Systematic inclusion of $\mathrm{CBC}$ parameters will help improve the reliability and sensitivity of PBS examinations.

\section{Authors' Disclosures of Potential Conflicts of Interest}

No potential conflicts of interest relevant to this article were reported.

\section{Acknowledgment}

This work was supported by the PBS Report Standardization Program of the Korean Society for Laboratory Hematology.

\section{REFERENCES}

1. Bain BJ. Diagnosis from the blood smear. N Engl J Med 2005;353:498507.

2. McPherson RA and Pincus MR. Henry's clinical diagnosis and management by laboratory methods. 23rd ed. Missouri, MO: Elsevier, 2017: 519-21.

3. Palmer L, Briggs C, McFadden S, Zini G, Burthem J, Rozenberg G, et al. ICSH recommendations for the standardization of nomenclature and grading of peripheral blood cell morphological features. Int J Lab Hematol 2015;37:287-303.

4. McFarlane A, Aslan B, Raby A, Bourner G, Padmore R. Critical values in hematology. Int J Lab Hematol 2015;37:36-43.

5. Vaughan J, Alli N, Mannaru K, Sedick Q. Refining peripheral blood smear review rules for neonatal inpatients in a South African academic laboratory. Int J Lab Hematol 2016;38:347-51.

6. Proytcheva MA. Issues in neonatal cellular analysis. Am J Clin Pathol 2009; 131:560-73.

7. Mayo Medical Laboratories, Pediatric test reference values http://www. mayomedicallaboratories.com/test-info/pediatric/refvalues/reference. php (Updated on Jun 2018).

8. Murari M and Pandey R. A synoptic reporting system for bone marrow aspiration and core biopsy specimens. Arch Pathol Lab Med 2006;130: 1825-9.

9. Mohanty SK, Piccoli AL, Devine LJ, Patel AA, William GC, Winters SB, et al. Synoptic tool for reporting of hematological and lymphoid neoplasms based on World Health Organization classification and College of American Pathologists checklist. BMC Cancer 2007;7:144.

10. Jaso J, Nguyen A, Nguyen AN. A synoptic reporting system for peripheral blood smear interpretation. Am J Clin Pathol 2011;135:358-64.

11. McFadden SL, Machin SJ, Simson E. The International Consensus Group for Hematology Review: suggested criteria for action following automated CBC and WBC differential analysis. Lab Hematol 2005;11: 83-90.

12. Nathan D and Oski F. Nathan and Oski's hematology of infancy and childhood. 7th ed. Philadelphia, PA: Saunders/Elsevier, 2009:1841-8.

13. Park SH, Park CJ, Lee BR, Kim MJ, Han MY, Cho YU, et al. Establishment of age-and gender-specific reference ranges for 36 routine and 57 cell population data items in a new automated blood cell analyzer, Sysmex XN-2000. Ann Lab Med 2016;36:244-9. 